\title{
Implementation, relevance, and virtual adaptation of neuro-oncological tumor boards during the COVID-19 pandemic: a nationwide provider survey
}

\author{
Niklas Schäfer ${ }^{1,2}$ - Elisabeth Bumes ${ }^{3} \cdot$ Fabian Eberle $^{4} \cdot$ Viola Fox $^{5} \cdot$ Florian Gessler $^{6} \cdot$ Frank A. Giordano $^{2,7}$. \\ Juergen Konczalla ${ }^{8}$. Julia Onken ${ }^{9,10,11}$ - Malte Ottenhausen ${ }^{12}$ - Moritz Scherer ${ }^{13}$ - Matthias Schneider ${ }^{2,14}$. \\ Hartmut Vatter ${ }^{2,14} \cdot$ Ulrich Herrlinger $^{1,2} \cdot$ Patrick Schuss ${ }^{2,14}$
}

Received: 21 May 2021 / Accepted: 3 June 2021 / Published online: 11 June 2021

(c) The Author(s) 2021

\begin{abstract}
Purpose Neuro-oncology tumor boards (NTBs) hold an established function in cancer care as multidisciplinary tumor boards. However, NTBs predominantly exist at academic and/or specialized centers. In addition to increasing centralization throughout the healthcare system, changes due to the COVID-19 pandemic have arguably resulted in advantages by conducting clinical meetings virtually. We therefore asked about the experience and acceptance of (virtualized) NTBs and their potential benefits.

Methods A survey questionnaire was developed and distributed via a web-based platform. Specialized neuro-oncological centers in Germany were identified based on the number of brain tumor cases treated in the respective institution per year. Only one representative per center was invited to participate in the survey. Questions targeted the structure/organization of NTBs as well as changes due to the COVID-19 pandemic.

Results A total of 65/97 institutions participated in the survey (response rate 67\%). In the context of the COVID-19 pandemic, regular conventions of NTBs were maintained by the respective centers and multi-specialty participation remained high. NTBs were considered valuable by respondents in achieving the most optimal therapy for the affected patient and in maintaining/encouraging interdisciplinary debate/exchange. The settings of NTBs have been adapted during the pandemic with the increased use of virtual technology. Virtual NTBs were found to be beneficial, yet administrative support is lacking in some places.

Conclusions Virtual implementation of NTBs was feasible and accepted in the centers surveyed. Therefore, successful implementation offers new avenues and may be pursued for networking between centers, thereby increasing coverage of neuro-oncology care.
\end{abstract}

Keywords Neuro-oncology · Tumor board · Virtualization · COVID-19

\section{Introduction}

Treatment of patients with cancers affecting the central and peripheral nervous system is complex and requires a coordinated team of specialists. Multidisciplinary tumor boards (MTBs) form the foundation for highly specialized (neuro)-oncology care and the continuous maintenance of the highest quality in cancer care [1]. The benefits of

Niklas Schäfer

niklas.schaefer@ukbonn.de

Extended author information available on the last page of the article
MTBs include efficient collaboration of multiple providers, communication between treatment teams, continuous education, increased adherence to treatment guidelines, and access to clinical trials [2,3]. However, MTBs not only provide an opportunity for consensus building on the best possible treatment regimens for individual patients, but also make a significant contribution to collegial adherence in individual case decisions. However, a study by Snyder et al. showed a high degree of heterogeneity in the implementation, proceeding, and documentation of such MTBs [4]. Going beyond the implementation of general MTB, Robin et al. recommend the establishment of a multidisciplinary brain tumor board led by neuro-oncologists 
for patient-centered neuro-oncology treatment planning and management to address the multiple demands in neuro-oncology patients. [5]. Appropriately, Snyder and colleagues state here that the implementation and delivery of neuro-oncology MTBs, including those at nonacademic centers, is critical for nationwide quality assurance of neuro-oncology patient care [4]. In addition, highly specialized neuro-oncology tumor boards (NTB) focusing solely on neuro-oncological patients cannot easily be established at every center. Nevertheless, the survival advantage of neuro-oncological patients treated at highvolume and/or academic centers seems evident $[6,7]$. The survey by Snyder et al. revealed that although the academic tumor centers polled had a desire to review external cases, only a quarter also experienced involvement from affiliated satellite centers [4]. To address the issue of nonparticipation of external centers, both teleconferencing options and increased use of virtual platforms have been proposed but rarely implemented to date [8, 9]. However, in the context of the COVID-19 pandemic, numerous efforts to increase digitalization/virtualization, particularly in healthcare, have accelerated [10,11]. This digitalization leap has not just created new challenges but, conversely, is also fostering new opportunities for expert networking in (neuro-) oncological tumor care [12].

The aim of the study was to identify the implementation modalities in academic and non-academic hospitals regarding NTBs in Germany in order to use this knowledge to further improve current practice and, if possible, to take advantage of the trends towards increasing digitalization.

\section{Methods}

\section{Survey population-neuro-oncological specialty centers}

The surveyed neuro-oncology centers in Germany were identified via Germany's leading provider transparency portal WeisseListe.de (WL.de). The portal WL.de has become the largest public portal for quality reports in healthcare in Germany [13]. The collected information originates from the statutory quality reports of over 2000 hospitals in Germany [14]. Since these quality reports are also based on the hospitals' accounting data, it was possible to record the number of reported brain tumor cases for each hospital using ICD-10 coding. For each hospital reporting more than 50 cases with ICD-10 code C71 (malignant disease of the brain) in a year, the authors' team manually identified one person responsible for neuro-oncology therapy using address lists. In this way, only one report per hospital took place with regard to the survey.

\section{Survey questionnaire}

Adhering to the design of a cross-sectional study, an online survey is created via a web-based platform (SurveyMonkey Inc.; San Mateo, California, USA; www.surveymonkey. com). The survey consisted of 24 questions formulated after discussion among the authors (supplementary appendix). The questions were grouped into 4 categories: (1) structure, (2) function/implementation, (3) changes due to the COVID-19 pandemic, and (4) impact of NTB on clinical practice. The questions on structure included information on the surveyed institutions and the format of NTB. Questions on the function/ implementation of the NTB addressed information on meeting tasks, activities, and staff composition as well as the respective implementation of the NTB in the participating centers. Changes caused by the COVID-19 pandemic were asked in a separate section (with an explicit focus on the expected increase in virtualization). Impact questions addressed the individual value of meetings as well as barriers. The survey was conclusively reviewed internally by a multidisciplinary group of physicians involved in neuro-oncology care at the University Hospital Bonn. The study was approved by the Institutional Review Board of the Medical Faculty of Bonn (no. 063/21).

\section{Data collection}

The survey was sent by email in March 2021 to the responsible medical staff involved in neuro-oncology at each hospital that had previously been identified using the described pathway. A total of 97 hospitals in Germany were contacted. No rewards or incentives for participation in the survey were offered, and those who refused to participate and/or did not complete the survey (more than three questions were missing) were considered non-responders. After one week, a reminder was sent in the same way to increase the response rate. Unique visitors were identified based on IP addresses and were used to prevent multiple entries from the same individual. The survey was available for a total duration of 3 weeks.

\section{Data analysis}

Survey responses were collected, downloaded, and converted into a dataset for further analysis. Summary statistics, simple and stratified, were compiled using SPSS (version 25, IBM Corp., Armonk, NY). 


\section{Results}

\section{Respondent characteristics}

Of the 97 centers invited, 65 (response rate $67 \%$ ) responded to this survey. With the exception of one federal state, $\geq 50 \%$ of centers per federal state in Germany were represented (Fig. 1). Of the responding centers, 53\% were a part of a university hospital, $29 \%$ were a part of a municipal hospital, and $18 \%$ were a part of a hospital with a private carrier. Overall, $5 \%$ of surveyed centers stated that they did not implement or were not affiliated with an NTB.

\section{Characteristics of the neuro-oncological tumor boards}

All centers that implemented NTB did so on a weekly schedule. The practice of conducting an NTB had been established for $>3$ years in $89 \%$ of the participating centers. $10 \%$ of centers had been conducting or participating in an NTB for 1-3 years, whereas $2 \%$ had been doing so for $<1$ year. Details on the participating specialties of NTBs are given in Table 1.

Regarding the diagnoses discussed in the NTB, all centers reported discussing patients with primary brain tumors $(100 \%)$. Brain metastases were also discussed in $95 \%$ of the centers and spinal tumor cases in $86 \% .47 \%$ of the participating centers also consulted on the treatment of paraneoplastic
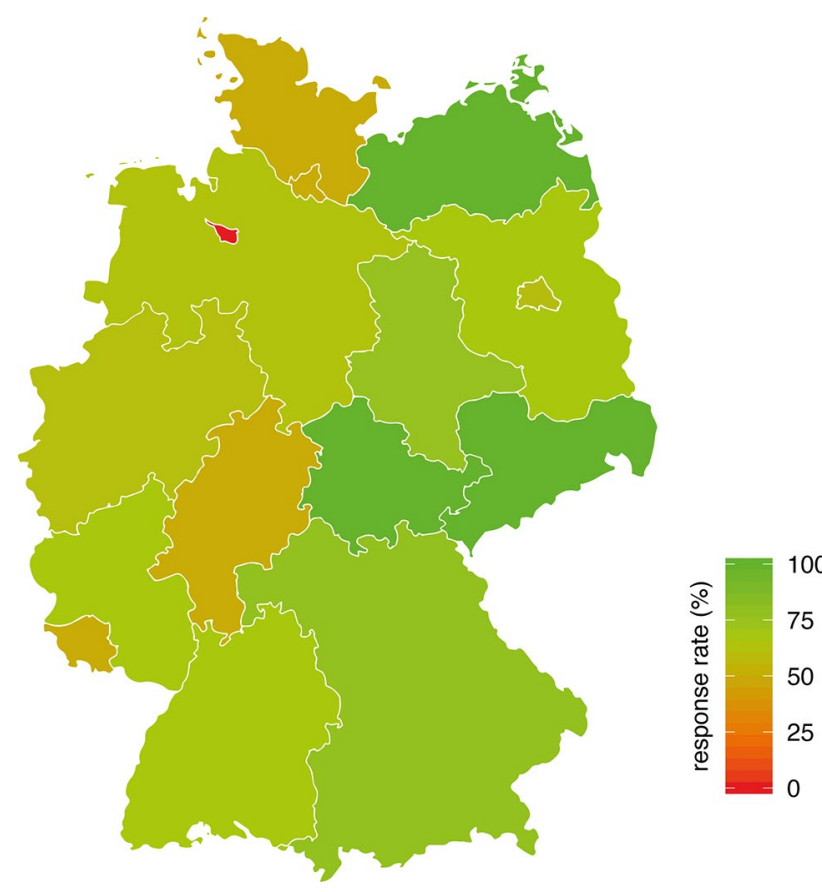

Fig. 1 Proportion of responding centers per federal state in Germany
Table 1 Participating specialties in neuro-oncological tumor boards

\begin{tabular}{lc}
\hline Participating disciplines & $(\%)$ \\
\hline Neurology/neuro-oncology & 77 \\
Neurosurgery & 98 \\
Radiation oncology & 89 \\
Medical oncology & 97 \\
(Neuro-) radiology & 98 \\
(Neuro-) pathology & 89 \\
Nuclear medicine & 25 \\
Dermatology & 15 \\
ENT/oral and maxillofacial surgery & 20 \\
Palliative medicine/care & 1 \\
Oncology nursing & 5 \\
Social worker & 2 \\
Study nurse/clinical trial personal & 14 \\
\hline
\end{tabular}

disease within their NTB. $86 \%$ reported to perform collaborative preliminary discussion of cases with lesions of unknown etiology. Presentation of the cases to be discussed is performed in $79 \%$ by the treating physician, in $56 \%$ also by a resident physician, and in only $5 \%$ by recitation of textonly information about the patient. $84 \%$ of responding centers reported receiving detailed information about patient comorbidities as part of the case presentation. In $82 \%$ of the cases, the NTBs also directly address the inclusion possibilities of potential clinical trials.

Case presentation includes active demonstration of radiological imaging in 98\%, and description of histological findings by (neuro)pathology in $82 \%$. In $89 \%$ of NTBs, the results of additional molecular pathology investigations are also part of the individual case discussion. $21 \%$ state that, especially in rare cases, a summary of the current research and/or a literature review is included in the case briefing. In $20 \%$, the demonstration of clinical cases is additionally complemented by histopathological images.

Documentation of NTB consultation results is done digitally in the patient's record in $91 \%$ of centers, including a separate report in $27 \%$ of cases, while in $9 \%$ of cases documentation is handled in the paper-based medical record. $73 \%$ of responding centers conduct regular morbidity and mortality (M\&M) conferences focusing on patient safety and quality improvement within their NTB, of which $88 \%$ do so at least twice a year.

\section{Changes during the COVID-19 pandemic}

The circumstances of the COVID-19 pandemic, including the contact restrictions required, have led to increased use of virtual technology in $68 \%$ of participating centers. $23 \%$ of centers are now operating their NTB completely virtually, while $48 \%$ are using a partially virtual environment but are 
responding to the pandemic's sanitation requirements with either larger locations, fewer people present, or increased sitting distance. The distribution of the utilized software for virtual implementation of NTB is as follows: $17 \%$ Zoom or Cisco Join/Webex, respectively, 13\% Skype for business, and $8 \%$ Microsoft Teams. The remaining used other or institutional solutions.

In free-text response options regarding subjective benefits of virtual NTB, the centers that have completed a virtual NTB conversion advocate improved integrability into daily clinical practice, significant time savings, better integration of external specialties/colleagues, higher numbers of participants, and significantly enhanced flexibility.

\section{Value of neuro-oncological tumor board}

All respondents consider the value of NTB to be the significant benefit of improving communication among medical colleagues (100\%). $88 \%$ consider the NTB as an opportunity to jointly achieve optimal/improved standardization and quality of care. $78 \%$ of the responding centers perceive an advantage in the continuous medical education stimulated by the NTB. $62 \%$ of the participants thought that the interdisciplinary meeting of NTB enables more treatment options for the individual patient. 50\% also regard the possibility of increased recruitment of patients for clinical neuro-oncological studies as an advantage of NTB.

The major obstacle to conducting a weekly NTB is perceived by $35 \%$ of respondents to be the high number of cases, while $25 \%$ consider it difficult to integrate the NTB into their daily clinical duties, $28 \%$ complain about a lack of support from the clinical administration and/or information technology (IT) services, whereas $27 \%$ identify the absence of individual specialty departments during the NTB and/ or within their center as a major constraint. According to respondents, $10 \%$ of centers experience scheduling conflicts between multiple MTBs. Within the scope of the free-text answers, complaints are found about the long duration of the weekly NTB, the resulting inconsistency with the stipulated work hours, and the sometimes deficient preparation/presentation of the cases to be discussed.

\section{Discussion}

Given the increased sub-specialization in the context of increasingly individualized tumor management, the establishment of specialized tumor boards (such as neuro-oncological tumor boards) seems worthwhile $[15,16]$. The respective benefits for both treatment and outcome of the patient, as well as the continuing education and training for the treating physicians along with the continuous selfreflection on applied treatment strategies in addition to the possibility of enrolling patients in clinical trials are proven advantages of NTBs $[4,17,18]$. Nevertheless, a continuous centralization of treatment can also be observed for neuro-oncological conditions, with a consequential reorganization of the hospital landscape towards the development of regional specialty centers/large-volume centers [19]. In addition to lower morbidity and mortality after (neuro) surgical procedures, improvements in overall survival have been demonstrated for various cancer types in large-volume centers due to the often improved inner-hospital multidisciplinary networking [7, 20-22]. Furthermore, corresponding accrediting authorities also set certain thresholds for the number of cases to be treated as part of the certification process for specialty cancer centers. To what extent this progressive centralization of neuro-oncology treatment can also ensure nationwide coverage is as yet unknown.

For this reason, our survey was addressed to German institutions with experience in neuro-oncology based on the number of diagnoses of malignant primary brain tumors per year. Given the thorough and stringent selection of survey participants, as well as the response rate of more than $60 \%$, we believe that the results presented here are robust for representative interpretation. Noteworthy, many of the respondents were non-academic institutions, mirroring a broad neuro-oncological care network in Germany given the low incidence rates of malignant primary brain tumors. Among the national facilities surveyed, NTBs are generally accepted and represent a worthwhile component of daily clinical routine in the view of the survey participants. Respondent institutions reported a high level of experience with NTBs, with $89 \%$ having established NTBs for more than 3 years. Only $5 \%$ of the responding centers have currently no NTBs implemented. This finding is underlined by the way cases are presented during these NTBs. The reported predominant participation and presentation by residents/treating physicians may indicate a true accountability of treating physicians and allows for detailed case discussions as well as study recruitment. Further, the estimated value of NTBs may increase willingness to participate. The vast majority rated the opportunities for communication, implementation of optimal patient care, and continuing medical education as beneficial. As expected, participation in NTBs is perceived to be time-consuming and may therefore interact with other duties in clinical practice.

\section{COVID-19 pandemic leading towards virtualization of NTBs}

The tremendous impact on daily living during the COVID19 pandemic resulted primarily in shifts in medical care [23-26]. However, the results of our survey impressingly demonstrate that NTBs were maintained during this period. This indicates the great efforts of local institutions and 
departments at this time, as well as the medical need for NTBs. Moreover, this occurred without a lower participation rate of individual disciplines, as could be demonstrated by the outstanding reported participation rate of neurosurgeons, radiation oncologists, medical oncologists and (neuro-) radiologists throughout this straining pandemic. A reason for this achievement may be the successful transition from face-to-face meetings to virtual meetings. This switch was reported as technically feasible and even resulted in a better integration of NTBs in daily practice routine. Interestingly, given the plethora of applicable software for virtual NTBs, the majority of institutions chose to use software from Zoom, Cisco, and Skype. In doing so, the virtual nature of the meetings seemed to facilitate the participation of the various disciplines, which would mean a significant benefit and increase in quality of NTBs held virtually. Another advantage of a virtual implementation of NTBs seems to be easier NTB participation of local and/or external guests, which allows for more in-depth individual case discussion and also cross-regional networking of all participating physicians [27]. Nevertheless, increasing virtualization also poses a challenge to strict compliance with data protection laws. Particularly in the case of cross-regional NTBs, this requires close coordination between the respective data protection officers in accordance with the prevailing federal state data protection laws.

\section{Value and relevance of NTBs}

The multidisciplinary approach in neuro-oncology implies non-delegable tasks of neurosurgeons and radiation oncologists as well as shared topics such as diagnostics and/or medical treatment, supportive and palliative care. Between these different disciplines, a leadership role for patients and caregivers is needed to ensure fixed contact partners and a managing/coordinating department. Especially patients with primary brain tumors as well as their caregivers might need a designated site for contact due to the high amount of psycho-social distress and impairment in physical and cognitive functioning [28]. Neuro-oncological neurologists, as representatives of a non-interventional discipline, may be predisposed to this role. As a noble goal, they might hold the reins, thereby coordinating necessary further medical/ surgical consultations and providing additional (neurologically skilled) supportive therapy to assist patients and their families throughout the course of their neuro-oncological disease. Obviously, these efforts and demand of great commitment could be supplanted by either one of the partnering disciplines. Nevertheless, only 4 of 5 participating institutions report that neurologists attend their NTBs, which makes increased enforcement within neuro-oncology by neurology specialists desirable given the medical care that must be provided to patients with tumors of the central nervous system.

Apart from patient management, the majority of respondents agree that the NTBs established at their centers contribute to improved physician communication, better interdisciplinary networking, and thus more optimal treatment of the patients entrusted to them. Resulting from the necessary contact restrictions in the context of the COVID-19 pandemic, the increased virtualization of NTBs promises to have a positive effect on improving attendance even for specialties not residing at the same hospital. Furthermore, due to the growing centralization in the healthcare system, a significantly improved integration of low-volume/remote hospitals might become feasible and thus contribute to a comprehensive, highly-specialized and optimal treatment of patients with CNS tumors.

\section{Limitations}

The main limitation of the present work that goes common to all surveys is the inability to generalize the results based on the selected surveyed sample. Due to the described selection procedure of the recipients of the survey, there is also a risk of selection bias. Nevertheless, the selection approach reduces the risk of multiple responses per center, and the overall survey coverage achieved is likely to reflect a reliable sentiment regarding the implementation of NTBs in Germany. Furthermore, the selection of one contact person per center naturally negates the discipline-specific characteristics, which, on the other hand, were not addressed by the survey itself.

\section{Conclusions}

Increasing centralization in the healthcare system also affects patients suffering from neuro-oncological tumors. The enormous efforts of healthcare providers in the context of the COVID-19 pandemic, including the augmented virtualization of neuro-oncological tumor boards, could help to implement optimal care for neuro-oncological patients even in remote hospitals and thus nationwide.

Supplementary Information The online version contains supplementary material available at https://doi.org/10.1007/s11060-021-03784-w.

Author contributions All authors contributed to the study conception and design. Material preparation was perforemd by all authors. Data collection and analysis were performed by NS and PS. The first draft of the manuscript was written by NS and PS and all authors commented on previous versions of the manuscript. All authors read and approved the final manuscript. 
Funding Open Access funding enabled and organized by Projekt DEAL. The authors did not receive support from any organization for the submitted work

Data availability The datasets generated during and/or analysed during the current study are available from the corresponding author on reasonable request.

Code availability A web-based platform was used provided by SurveyMonkey Inc. (San Mateo, California, USA; www.surveymonkey.com). For data analysis we used SPSS version 25 (IBM Corp., Armonk, NY).

\section{Declarations}

Conflict of interest The authors declare that the article content was composed in the absence of any commercial or financial relationship that could be construed as a potential conflict of interest.

Ethical approval The study was approved by the Institutional Review Board of the Medical Faculty of Bonn (no. 063/21).

Open Access This article is licensed under a Creative Commons Attribution 4.0 International License, which permits use, sharing, adaptation, distribution and reproduction in any medium or format, as long as you give appropriate credit to the original author(s) and the source, provide a link to the Creative Commons licence, and indicate if changes were made. The images or other third party material in this article are included in the article's Creative Commons licence, unless indicated otherwise in a credit line to the material. If material is not included in the article's Creative Commons licence and your intended use is not permitted by statutory regulation or exceeds the permitted use, you will need to obtain permission directly from the copyright holder. To view a copy of this licence, visit http://creativecommons.org/licenses/by/4.0/.

\section{References}

1. American Society of Clinical O, European Society for Medical O (2006) Asco-esmo consensus statement on quality cancer care. Ann Oncol 17(7):1063-1064

2. Lamb BW, Sevdalis N, Arora S, Pinto A, Vincent C, Green JS (2011) Teamwork and team decision-making at multidisciplinary cancer conferences: barriers, facilitators, and opportunities for improvement. World J Surg 35(9):1970-1976

3. Wright FC, De Vito C, Langer B, Hunter A, Expert Panel on Multidisciplinary Cancer Conference S (2007) Multidisciplinary cancer conferences: a systematic review and development of practice standards. Eur J Cancer 43(6):1002-1010

4. Snyder J, Schultz L, Walbert T (2017) The role of tumor board conferences in neuro-oncology: a nationwide provider survey. $\mathrm{J}$ Neurooncol 133(1):1-7

5. Robin AM, Walbert T, Mikkelsen T, Kalkanis SN, Rock J, Lee I, Rosenblum ML (2014) Through the patient's eyes: the value of a comprehensive brain tumor center. J Neurooncol 119(3):465-472

6. Koshy M, Sher DJ, Spiotto M, Husain Z, Engelhard H, Slavin K, Nicholas MK, Weichselbaum RR, Rusthoven C (2017) Association between hospital volume and receipt of treatment and survival in patients with glioblastoma. J Neurooncol 135(3):529-534

7. Zhu P, Du XL, Zhu JJ, Esquenazi Y (2019) Improved survival of glioblastoma patients treated at academic and high-volume facilities: a hospital-based study from the national cancer database. J Neurosurg 132(2):491-502

8. Augestad KM, Lindsetmo RO (2009) Overcoming distance: video-conferencing as a clinical and educational tool among surgeons. World J Surg 33(7):1356-1365

9. Munro AJ, Swartzman S (2013) What is a virtual multidisciplinary team (vMDT)? Br J Cancer 108(12):2433-2441

10. Keesara S, Jonas A, Schulman K (2020) Covid-19 and health care's digital revolution. N Engl J Med 382(23):e82

11. Temesgen ZM, DeSimone DC, Mahmood M, Libertin CR, Varatharaj Palraj BR, Berbari EF (2020) Health care after the COVID19 pandemic and the influence of telemedicine. Mayo Clin Proc 95(9S):S66-S68

12. Dharmarajan H, Anderson JL, Kim S, Sridharan S, Duvvuri U, Ferris RL, Solari MG, Clump DA 2nd, Skinner HD, Ohr JP, Zandberg DP, Bt B, Hughes MA, Traylor KS, Seethala R, Chiosea SI, Nilsen ML, Johnson JT, Kubik MW (2020) Transition to a virtual multidisciplinary tumor board during the covid-19 pandemic: university of pittsburgh experience. Head Neck 42(6):1310-1316

13. Pross C, Averdunk LH, Stjepanovic J, Busse R, Geissler A (2017) Health care public reporting utilization-user clusters, web trails, and usage barriers on Germany's public reporting portal Weisse-Liste. De. BMC Med Inform Decis Mak 17(1):48

14. Emmert M, Hessemer S, Meszmer N, Sander U (2014) Do German hospital report cards have the potential to improve the quality of care? Health Policy 118(3):386-395

15. Field KM, Rosenthal MA, Dimou J, Fleet M, Gibbs P, Drummond K (2010) Communication in and clinician satisfaction with multidisciplinary team meetings in neuro-oncology. J Clin Neurosci 17(9):1130-1135

16. Horbinski C, Ligon KL, Brastianos P, Huse JT, Venere M, Chang S, Buckner J, Cloughesy T, Jenkins RB, Giannini C, Stupp R, Nabors LB, Wen PY, Aldape KJ, Lukas RV, Galanis E, Eberhart CG, Brat DJ, Sarkaria JN (2019) The medical necessity of advanced molecular testing in the diagnosis and treatment of brain tumor patients. Neuro Oncol 21(12):1498-1508

17. Lutterbach J, Pagenstecher A, Spreer J, Hetzel A, Velthoven V, Nikkhah G, Frommhold H, Volk B, Schumacher M, Lucking C, Zentner J, Ostertag C (2005) The brain tumor board: lessons to be learned from an interdisciplinary conference. Onkologie 28(1):22-26

18. Ameratunga M, Miller D, Ng W, Wada M, Gonzalvo A, Cher L, Gan HK (2018) A single-institution prospective evaluation of a neuro-oncology multidisciplinary team meeting. J Clin Neurosci $56: 127-130$

19. Barker FG 2nd, Curry WT Jr, Carter BS (2005) Surgery for primary supratentorial brain tumors in the United States, 1988 to 2000: the effect of provider caseload and centralization of care. Neuro Oncol 7(1):49-63

20. Aulakh S, DeDeo MR, Free J, Rosenfeld SS, Quinones-Hinojosa A, Paulus A, Manna A, Manochakian R, Chanan-Khan AA, Ailawadhi S (2019) Survival trends in glioblastoma and association with treating facility volume. J Clin Neurosci 68:271-274

21. Ajmani GS, James TA, Kantor O, Wang CH, Yao KA (2017) The impact of facility volume on rates of pathologic complete response to neoadjuvant chemotherapy used in breast cancer. Ann Surg Oncol 24(11):3157-3166

22. Wouters MW, Wijnhoven BP, Karim-Kos HE, Blaauwgeers HG, Stassen LP, Steup WH, Tilanus HW, Tollenaar RA (2008) Highvolume versus low-volume for esophageal resections for cancer: the essential role of case-mix adjustments based on clinical data. Ann Surg Oncol 15(1):80-87

23. Mohile NA, Blakeley JO, Gatson NTN, Hottinger AF, Lassman AB, Ney DE, Olar A, Schiff D, Shih HA, Strowd R, van den Bent MJ, Ziu M (2020) Urgent considerations for the 
neuro-oncologic treatment of patients with gliomas during the covid-19 pandemic. Neuro Oncol 22(7):912-917

24. Gessler F, Lehmann F, Bösel J, Fuhrer H, Neugebauer H, Wartenberg KE, Wolf S, Bernstock JD, Niesen WD, Schuss P (2020) Triage and allocation of neurocritical care resources during the COVID 19 pandemic — a national survey. Front Neurol 11:609227

25. Ramakrishna R, Zadeh G, Sheehan JP, Aghi MK (2020) Inpatient and outpatient case prioritization for patients with neuro-oncologic disease amid the COVID-19 pandemic: general guidance for neuro-oncology practitioners from the aans/cns tumor section and society for neuro-oncology. J Neuro-Oncol 147(3):525-529

26. Bernhardt D, Wick W, Weiss SE, Sahgal A, Lo SS, Suh JH, Chang EL, Foote M, Perry J, Meyer B, Vajkoczy P, Wen PY, Straube C, Pigorsch S, Wilkens JJ, Combs SE (2020) Neuro-oncology management during the COVID-19 pandemic with a focus on WHO grade iii and iv gliomas. Neuro Oncol 22(7):928-935
27. Daggubati LC, Eichberg DG, Ivan ME, Hanft S, Mansouri A, Komotar RJ, D'Amico RS, Zacharia BE (2020) Telemedicine for outpatient neurosurgical oncology care: lessons learned for the future during the COVID-19 pandemic. World Neurosurg 139:e859-e863

28. Coomans MB, Dirven L, Aaronson NK, Baumert BG, Van Den Bent M, Bottomley A, Brandes AA, Chinot O, Coens C, Gorlia T, Herrlinger U, Keime-Guibert F, Malmstrom A, Martinelli F, Stupp R, Talacchi A, Weller M, Wick W, Reijneveld JC, Taphoorn MJB (2019) Symptom clusters in newly diagnosed glioma patients: which symptom clusters are independently associated with functioning and global health status? Neuro Oncol 21(11):1447-1457

Publisher's Note Springer Nature remains neutral with regard to jurisdictional claims in published maps and institutional affiliations.

\section{Authors and Affiliations}

\section{Niklas Schäfer ${ }^{1,2}$ (1) Elisabeth Bumes ${ }^{3} \cdot$ Fabian Eberle $^{4} \cdot$ Viola Fox $^{5} \cdot$ Florian Gessler $^{6} \cdot$ Frank A. Giordano $^{2,7}$. Juergen Konczalla ${ }^{8}$. Julia Onken ${ }^{9,10,11} \cdot$ Malte Ottenhausen $^{12} \cdot$ Moritz Scherer $^{13} \cdot$ Matthias Schneider $^{2,14}$. Hartmut Vatter $^{2,14}$. Ulrich Herrlinger ${ }^{1,2}$. Patrick Schuss ${ }^{2,14}$}

1 Division of Clinical Neuro-Oncology, Department of Neurology, University Hospital Bonn, Venusberg-Campus 1, 53127 Bonn, Germany

2 Neuro-Oncology Center, Center of Integrated Oncology (CIO) Bonn, University Hospital Bonn, Bonn, Germany

3 Department of Neurology and Wilhelm Sander-NeuroOncology Unit, Regensburg University Hospital, Regensburg, Germany

4 Department of Radiation Oncology, University Hospital Marburg, Marburg, Germany

5 Lung Cancer Center Köln-Merheim, Cologne, Germany

6 Department of Neurosurgery, University Medicine Rostock, Rostock, Germany

7 Department of Radiotherapy and Radiation Oncology, University Hospital Bonn, Bonn, Germany
8 Department of Neurosurgery, University Hospital Frankfurt, Frankfurt, Germany

9 Department of Neurosurgery, Charité-Universitätsmedizin Berlin, corporate member of Freie Universität Berlin, Humboldt-Universität Zu Berlin, Berlin, Germany

10 Berlin Institute of Health, Berlin, Germany

11 German Cancer Consortium (DKTK), Partner Site Berlin, CCCC (Campus Mitte), Berlin, Germany

12 Department of Neurosurgery, University Hospital Mainz, Mainz, Germany

13 Department of Neurosurgery, University Hospital Heidelberg, Heidelberg, Germany

14 Department of Neurosurgery, University Hospital Bonn, Bonn, Germany 